\title{
Análisis de concordancia de atributos en color de piezas galvanizadas
}

\section{Color attribute agreement analysis of galvanized pieces}

\author{
SALAS DOMINGUEZ, Mario Iván† \& MUÑOZ DÍAZ, Ismael \\ Universidad Tecnológica de Aguascalientes \\ ID $1^{\text {er }}$ Autor: Mario Iván, Salas-Dominguez / ORC ID: 0000-0001-7609-3109, CVU CONACYT ID: 534378 \\ ID $1^{\text {er }}$ Coautor: Ismael, Muñoz-Díaz / ORC ID: 0000-0003-2779-8932, CVU CONACYT ID: 998197
}

DOI: $10.35429 /$ JTIP.2019.6.3.1.6

Recibido 03 de Enero, 2019; Aceptado 09 Marzo, 2019

\section{Resumen}

El presente trabajo tiene como objetivo establecer la confiabilidad del sistema medición por atributos en la característica de color para las piezas galvanizadas como producto final. Se evaluaron 6 operadores con 30 muestras de piezas metálicas galvanizadas roladas en frío y en caliente en una proporción de $50 \%$ de piezas aceptadas y $50 \%$ rechazadas establecido por el personal de calidad de la planta de Techengineering en la ciudad de Aguascalientes, Aguascalientes, bajo un criterio de pasa-no pasa. Para llevar a cabo el estudio se utilizó el programa Minitab®16 usando la herramienta análisis de concordancia. De acuerdo a los coeficientes Kappa de Fleiss e intervalos de confianza generados en el reporte se pudo establecer que la precisión del sistema se califica entre excelente y buena y la exactitud como buena de forma marginal, con esta información se logró establecer las estrategias para mejorar el sistema de medición.

ASM, Coeficiente Kappa de Fleiss

\begin{abstract}
The objective of this work is to establish the reliability of the measurement system by attributes in the color characteristic for the galvanized parts as final product. Six operators were evaluated with 30 samples of galvanized metal parts cold and hot rolled in a proportion of $50 \%$ of accepted parts and $50 \%$ rejected by the quality staff of the Techengineering plant in the city of Aguascalientes, Aguascalientes, under a criterion of passes-does not pass. To carry out the study, the attribute agreement analysis tool of Minitab®16 was used. According to the Fleiss Kappa coefficient and confidence intervals generated in the report, it was established that the accuracy of the system is valued between excellent and good and the accuracy as good marginally, with this information it was possible to establish strategies to improve the measurement system.
\end{abstract}

Tags MSA, Fleiss Kappa coefficient

Citación: SALAS DOMINGUEZ, Mario Iván \& MUÑOZ DÍAZ, Ismael. Análisis de concordancia de atributos en color de piezas galvanizadas. Revista de Tecnologías en Procesos Industriales. 2019. 3-6: 1-6

\footnotetext{
* Correspondencia del Autor (msalas@utags.edu.mx)

$\dagger$ Investigador contribuyendo como primer autor.
} 


\section{Introducción}

El presente estudio tiene como origen un proyecto de Seis Sigma en la planta de TechEngineering en la ciudad de Aguascalientes, Aguascalientes para disminuir el número de rechazos de piezas galvanizadas por la característica de color. La metodología Seis Sigma destaca en su segunda etapa, la validación del sistema de medición y esto está sustentado en el hecho de que en todo proceso productivo es importante garantizar la confiabilidad del sistema de medición antes de actuar sobre el proceso y tomar acciones erróneas debidas a datos falsos.

El presente trabajo tiene como objetivo evaluar el sistema de medición por atributos del color de las placas galvanizadas empleando la herramienta de análisis de concordancia por atributos en Minitab 16®.

En la metodología a desarrollar se explica qué es lo que se debe de analizar en un sistema de medición, la justificación de los principios estadísticos a utilizar en base al tipo de dato que se usará en el estudio, la interpretación que deberá de realizarse a los índices logrados y las condiciones recomendables bajo las cuales debe de realizarse el estudio.

En la sección del desarrollo, se exponen las condiciones bajo las cuales se llevó a cabo el estudio desde el punto de vista operativo, así como los comandos empleados en Minitab®. En la sección de resultados se presentan los índices estadísticos y gráficos que arroja Minitab® y se lleva a cabo una discusión de ellos.

Por último, en las conclusiones, se explican las repercusiones que tienen los resultados obtenidos así como las acciones recomendadas para la mejora del sistema de medición.

\section{Metodología a desarrollar}

La confiabilidad del sistema de medición debe de ser evaluada tanto en su precisión como en la exactitud. La precisión de un sistema de medición hace referencia a la variación de las medidas tomadas por un operador como la variación que existe entre los operadores y la exactitud es a la cercanía de las evaluaciones realizadas con respecto a un patrón, estándar o referencia.
Un sistema de medición puede clasificarse en base al tipo de dato que maneja como de variable o atributo, para el caso que se estudió el cual consistió de una evaluación de aceptar o rechazar piezas metálicas galvanizadas por su apariencia de color con respecto a un estándar cae en la clasificación de un sistema de medición por atributos del tipo binario.

Para cubrir esta serie de requerimientos se realizó un análisis de concordancia de atributos mediante el programa Minitab $16 \AA$. (Minitab, s.f.)

Dentro del análisis de concordancia, Minitab® presenta las opciones del uso de los índices Kappa de Fleiss, Cohen o el coeficiente de Kendall. El coeficiente de Kendall es usado para variables por atributos del tipo ordinal (Picado Alvarado, Federico, 2008) mientras que el índice Kappa es usado para sistemas de evaluación cualitativos por atributos binarios del tipo pasa o no pasa (Romero \& Romero, 2011) El índice Kappa mejor conocido como Kappa de Cohen es un valor que busca establecer el acuerdo que existe entre diferentes evaluadores o con respecto a un estándar despreciando el factor aleatorio, la fórmula es la siguiente (Servizo Galego de Saúde, 2014).

$\kappa=\frac{\operatorname{Pr}(a)-\operatorname{Pr}(e)}{1-\operatorname{Pr}(e)}$

Donde:

$\kappa$ Índice Kappa

$\operatorname{Pr}$ (a) Acuerdo relativo entre los operadores

$\operatorname{Pr}(\mathrm{e})$ Probabilidad de acuerdo por el azar.

Los valores de Kappa van de -1 a 1 interpretándose de la siguiente forma (Cerda \& Villarroel, 2008)

Un valor de kappa de 1 indica que existe una alta concordancia entre los evaluadores y esta no se debe al azar.

Un valor de kappa de 0 indica que el acuerdo entre operadores se debe al azar.

Un valor de kappa menos de 1 manifiesta desacuerdo entre los operadores. 
Los valores que se buscan deberán de estar entre 0 y 1 sin embargo que tan cerca de 1 se puede considerar correcto, esto es difícil de llegar a un acuerdo por lo que se debe de tener en cuenta el contexto en el que se realiza el estudio,( Landis y Koch 1977, citado por Servizo Galego de Saúde, 2014) proponen "una escala de interpretación del valor de kappa que considera como aceptable un valor mayor o igual a 0,40 y excelentes los valores superiores a 0,75 ", similar a (AUTOMOTIVE INDUSTRY ACTION GROUP (AIAG), 2010) que proponen "valores de kappa mayores que 0.75 indican una concordancia de buena a excelente (con un Kappa máximo $=1$ ) los valores menores que 0.40 indican poca concordancia”, (Altman DG., citado por López de Ullibarri \& Pita Fernández, 1999) proponen como criterio de evaluación del índice kappa la Tabla 1.

\begin{tabular}{|c|c|}
\hline Valor de $\mathbf{k}$ & Fuerza de la concordancia \\
\hline$<0.20$ & Pobre \\
\hline $0.21-0.40$ & Débil \\
\hline $0.41-0.60$ & Moderada \\
\hline $0.61-0.80$ & Buena \\
\hline $0.81-1.00$ & Muy buena \\
\hline
\end{tabular}

Tabla 1 Valoración del índice kappa

Adicional al Kappa de Cohen existe el Kappa de Fleiss, para el estudio se usó este último en base a la recomendación de (Minitab, s.f.) "El kappa de Cohen es un estadístico que suele utilizarse para medir la concordancia en las evaluaciones de dos evaluadores. El kappa de Fleiss es una generalización del kappa de Cohen para más de 2 evaluadores." Esta afirmación también es reiterada por (Cerda \& Villarroel, 2008)

Para un análisis de concordancia por atributos Minitab ${ }^{\circledR}$ evalúa tanto la exactitud como la precisión para el operador como entre operadores, proporcionando los porcentajes de coincidencia, así como los estadísticos kappa y sus correspondientes $\mathrm{p}$ valores, útiles para poder establecer la inferencia estadística al considera la hipótesis nula como una coincidencia debida al azar y la alternativa como una coincidencia debido a las habilidades de los evaluadores y no al azar.(Minitab INC., 2019b) Para el desarrollo del análisis del sistema de medición por atributos (Minitab INC., 2019a)da las siguientes recomendaciones.

1. Las evaluaciones que realizan los inspectores deben de ser de forma aleatoria.
2. Es viable comparar contra una referencia.

3. Para obtener estimaciones adecuadas emplear un mínimo de 50 muestras abarcando todo el rango posible de valores y dar preferencia a más muestras que al número de réplicas.

4. Se debe de evaluar al menos dos veces cada muestra por cada inspector.

5. $\mathrm{Al}$ menos 3 inspectores abarcando todos los que realizan la inspección incluyendo hábiles y novatos.

6. Cada inspector debe de evaluar el mismo número de muestras y replicas.

\section{Desarrollo}

Para llevar a cabo el estudio se seleccionaron a todos los operadores del proceso ya que todos estaban facultados para calificar el color del producto final. Previo al estudio se definió por parte del departamento de control de calidad el rango del color estándar correcto tanto para piezas roladas en frio como en caliente y se dio información a los operadores sobre estos estándares, adicional se implementó una caja negra para facilitar su evaluación.

Se elaboraron 30 muestras a ser evaluadas con una proporción de aceptación del $50 \%$, situación similar se presenta en el muestreo de piezas que presenta el estudio de (Romero \& Romero, 2011) con 30 piezas en una proporción de 17 piezas buenas y el resto piezas malas.

Cada una de las muestras fue identificada con un número, así como los operadores. Para garantizar la aleatoriedad del proceso se generó una tabla usando Minitab ${ }^{\circledR}$ con la secuencia Estadísticas $>$ Herramientas de calidad $>$ Crear hoja de trabajo de análisis de concordancia.

La configuración de la hoja de trabajo quedo de la siguiente forma.

\begin{tabular}{|l|l|}
\hline \multicolumn{1}{|c|}{ Elemento } & \multicolumn{1}{c|}{ Valor } \\
\hline Número de operadores & 6 \\
\hline Numero de muestras & 30 \\
\hline Numero de replicas & 2 \\
\hline Estándar aceptado & ok \\
\hline Estándar rechazado & $\mathrm{X}$ \\
\hline
\end{tabular}

Tabla 2 Parámetros de configuración hoja de muestreo 
Con esto Minitab® generó una hoja de registro para la calificación de las muestras en orden aleatorio, así como el orden en que los operadores pasan a calificar las muestras.

La prueba se realizó con cinco horas de diferencia entre una réplica y otra esto con el fin de evitar el efecto de recordar la calificación de la pieza. Los datos recopilados se capturaron en la hoja de trabajo de Minitab® generada anteriormente y para procesar la información se ejecutó la secuencia de comandos Estadísticas > Herramientas de calidad > Análisis de concordancia de atributos para poder obtener los valores de los índices kappa y la opción Asistente>Análisis del sistema de medición (MSA)>Análisis de concordancia de atributos para poder obtener el reporte detallado de exactitud y el de clasificación incorrecta.

\section{Resultados}

\section{Análisis de precisión}

El análisis de precisión se realiza evaluando las coincidencias que el evaluador tiene consigo mismo, así como con respecto a los otros evaluadores.

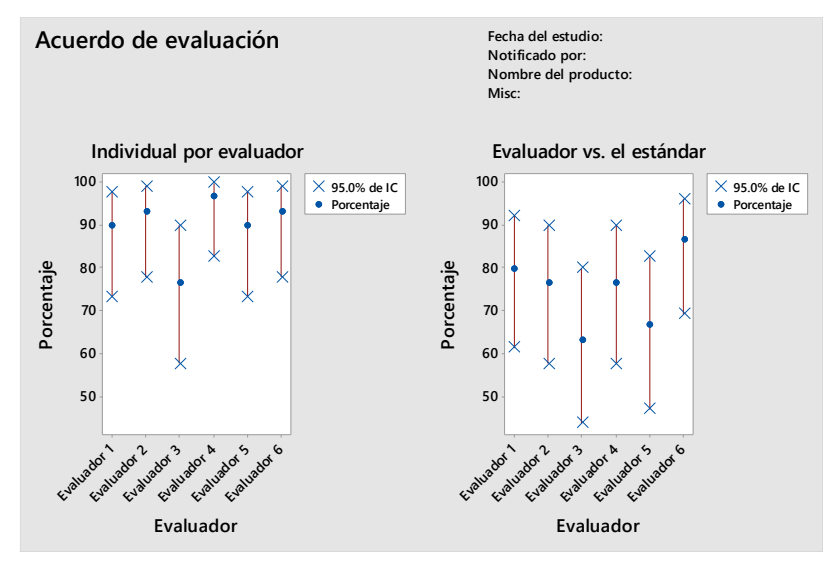

Gráfico 1 Acuerdo de evaluación

\begin{tabular}{|l|c|c|c|c|}
\hline Evaluador & Kappa & \multicolumn{1}{c|}{$\begin{array}{c}\text { Error } \\
\text { estándar de } \\
\text { Kappa }\end{array}$} & $\begin{array}{c}\text { P(vs > } \\
\text { 0) }\end{array}$ \\
\hline Evaluador 1 & 0.798 & 0.183 & 4.371 & 0.000 \\
\hline Evaluador 2 & 0.861 & 0.183 & 4.717 & 0.000 \\
\hline Evaluador 3 & 0.520 & 0.183 & 2.848 & 0.002 \\
\hline Evaluador 4 & 0.923 & 0.183 & 5.055 & 0.000 \\
\hline Evaluador 5 & 0.769 & 0.183 & 4.212 & 0.000 \\
\hline Evaluador 6 & 0.861 & 0.183 & 4.717 & 0.000 \\
\hline
\end{tabular}

Tabla 3 Índice Kappa de Fleiss acuerdo del evaluador consigo mismo
De la Tabla 3 Los p valores de todos los evaluadores son inferiores de 0.05 lo que nos proporciona evidencia estadística para poder rechazar la hipótesis nula y considerar que la coincidencia en cada evaluador no es debida al azar con un nivel de significancia del $5 \%$.

Esta misma tabla muestra que la concordancia del evaluador consigo mismo es de buena a excelente como lo propone AIAG esto se observa en los valores de kappa superiores a 0.75 en todos los evaluadores a excepción del evaluador 3 .

Esta conclusión también se ve reforzada observando el

Gráfico 1 Acuerdo de evaluación en donde se puede observar en el gráfico individual por evaluador en donde se muestra el intervalo de confianza del $95 \%$ del porcentaje de acuerdo para cada evaluador y se observa el nivel superior al $90 \%$ excepto el evaluador 3 .

\begin{tabular}{|c|c|c|c|}
\hline Kappa & \multicolumn{1}{c}{$\begin{array}{c}\text { Error estándar de } \\
\text { Kappa }\end{array}$} & Z & $\begin{array}{c}\text { P(vs > } \\
\mathbf{0})\end{array}$ \\
\hline 0.7137 & 0.02247 & 31.7579 & 0.0000 \\
\hline
\end{tabular}

Tabla 4 Índice kappa de Fleiss acuerdo entre evaluadores

La Tabla 4, nos da información sobre el nivel de acuerdo entre los 6 evaluadores, en este caso el valor de $\mathrm{p}$ nos permite rechazar la hipótesis nula y afirmar que la coincidencia entre los operadores no se debe al azar con un nivel de significancia del $5 \%$

El valor kappa de 0.71 nos indica que es aceptable y muy cerca de considerarse buena a los criterios de AIAG, y en base a los valores de la Tabla 1, podemos decir que el nivel es bueno.

\section{Análisis de exactitud}

El análisis de exactitud busca evaluar el nivel de acuerdo que tienen los operadores con respecto al valor estándar establecido.

\begin{tabular}{|r|r|c|r|}
\hline Kappa & Error estándar de Kappa & $\mathrm{Z}$ & $\mathrm{P}(\mathrm{vs}>0)$ \\
\hline 0.592 & 0.053 & 11.238 & 0.000 \\
\hline
\end{tabular}

Tabla 5 Índice kappa de Fleiss todos los valuadores vs. el estándar 


\begin{tabular}{|l|l|l|l|l|}
\hline \multicolumn{1}{|c|}{ Evaluador } & Kappa & $\begin{array}{l}\text { Error estánd } \\
\text { ar de Kappa }\end{array}$ & \multicolumn{1}{c|}{ P(vs > 0 } \\
\hline Evaluador 1 & 0.698 & 0.129 & 5.409 & 0.000 \\
\hline Evaluador 2 & 0.596 & 0.129 & 4.616 & 0.000 \\
\hline Evaluador 3 & 0.497 & 0.129 & 3.848 & 0.000 \\
\hline Evaluador 4 & 0.552 & 0.129 & 4.273 & 0.000 \\
\hline Evaluador 5 & 0.414 & 0.129 & 3.204 & 0.001 \\
\hline Evaluador 6 & 0.797 & 0.129 & 6.176 & 0.000 \\
\hline
\end{tabular}

Tabla 6 Índice kappa de Fleiss evaluador vs estándar

Por lo que respecta a la exactitud Tabla 5 el valor p menor a 0.05 nos permite afirmar la significancia estadística de que no influye el componente aleatorio, el valor de kappa de 0.59 es aceptable, sin embargo aún dista de ser bueno para AIAG, en base a la tabla

Revisando la Tabla 6, vemos que los valores del índice kappa están por debajo de 0.6 a excepción de los evaluadores 1 y 7 con un índice de 0.7 o más como el caso del evaluador 6.

El Grafico 1, en el diagrama evaluador vs. el estándar en donde se observan los intervalos de confianza para cada evaluador del porcentaje de acuerdo vs el estándar se identifica al evaluador 6 con el mayor porcentaje y al evaluador 3 con el menor porcentaje.

Para profundizar en el análisis de la exactitud se genera un reporte detallado que se muestra en el Grafico 2 y Grafico 3.

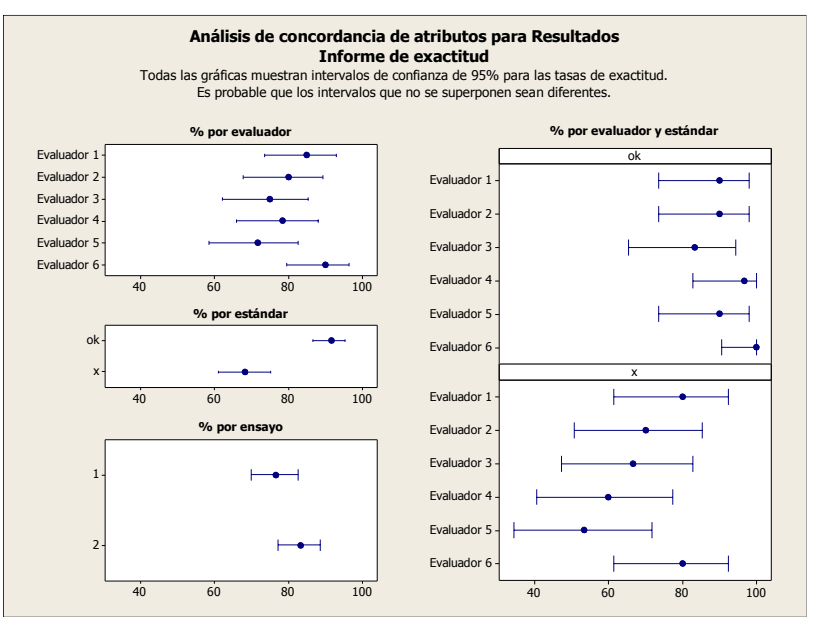

Gráfico 2 Intervalos de confianza de la exactitud

En el Grafico 2 en el diagrama \% por estándar, podemos ver que los intervalos de confianza no se traslapan, por lo que es prudente afirmar que existe una diferencia en el criterio para calificar piezas buenas y malas, en este caso se califican mejor las piezas buenas que las malas.
En el mismo Gráfico en el diagrama de $\%$ por evaluador y estándar observamos como los evaluadores 6 y después el 1 muestran la mejor habilidad al momento de calificar las piezas malas, este comportamiento también lo vemos en el Grafico 3, gráfico x calificados como ok que viene a ser el complemento.

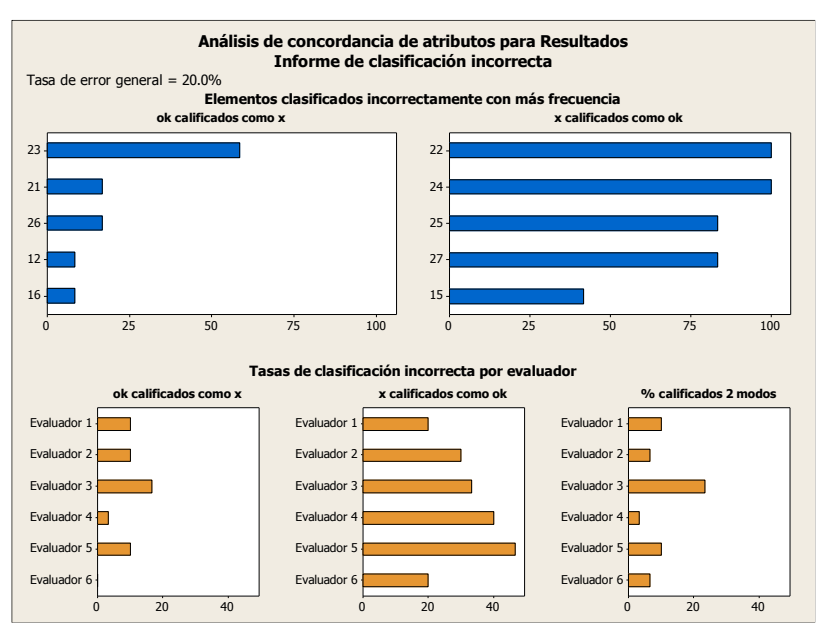

Gráfico 3 Informe de clasificaciones incorrectas

Del Grafico 3, podemos ver en el diagrama $\mathrm{x}$ calificados como ok, esto es piezas malas calificadas como buenas, las piezas que tienen un alto índice de calificación incorrecta son la 22,24,25,27. Una revisión de estas piezas nos indicó que corresponden a piezas roladas en caliente lo que indica la dificultad del operador para calificar el color con esta característica de la materia prima.

\section{Conclusiones}

Con el análisis de la información podemos concluir en base a los índices kappa que la precisión del sistema es adecuada ya que el acuerdo en el operador la calificamos como excelente y el acuerdo entre operadores como aceptable y muy cerca del nivel de bueno para AIAG. En lo que respecta a la exactitud esta es aceptable pero aún dista de ser buena por lo que en este rubro es en donde se deben de aplicar las primeras acciones correctivas de capacitación en el personal que evalúa las piezas. 
En la capacitación se debe de hacer especial énfasis en el criterio de aquellas piezas que sean roladas en caliente y no cumplan el estándar. En base a los resultados podemos identificar que el evaluador 6 es prospecto para convertirse en un capacitador por su excelente desempeño y la misma información nos indica la necesidad de capacitación a la brevedad para los evaluadores 2 al 5 que muestran área de oportunidad incrementándose en el orden antes mencionado como lo muestra el Grafico 3, el diagrama $\%$ por evaluador y estándar el correspondiente a $\mathrm{x}$.

Adicional el evaluador 3 deberá de afinarse su consistencia en las evaluaciones que realiza.

\section{Referencias}

\section{AUTOMOTIVE INDUSTRY ACTION GROUP (AIAG). (2010). Measurement System Analysis, Reference Manual (4ta ed.).}

Cerda, J., \& Villarroel, L. (2008). Evaluación de la concordancia inter-observador en investigación pediátrica: Coeficiente de Kappa. Revista Chilena de Pediatria, 79(1), 54-58.

López de Ullibarri, G., \& Pita Fernández, S. (1999). Medidas de concordancia: el índice de Kappa. Cad Aten Primaria, (6), 169-171. recuperado de www.fisterra.com

Minitab INC. (2019a). Consideraciones acerca de los datos para Análisis de concordancia de atributos. recuperado agosto 18, 2019, de https://support.minitab.com/es$\mathrm{mx} / \mathrm{minitab} / 18 /$ help-and-how-to/quality-andprocess-improvement/measurement-systemanalysis/how-to/attribute-agreementanalysis/attribute-agreement-analysis/beforeyou-start/data-considerations/

Minitab INC. (2019b). Hipótesis para Análisis de concordancia de atributos. recuperado agosto 17, 2019, de https://support.minitab.com/es$\mathrm{mx} / \mathrm{minitab} / 18 /$ help-and-how-to/quality-andprocess-improvement/measurement-systemanalysis/how-to/attribute-agreementanalysis/attribute-agreement-analysis/beforeyou-start/hypotheses/
Minitab INC. (2019c). Revisión general de Análisis de concordancia de atributos. recuperado agosto 15, 2019, de https://support.minitab.com/es$\mathrm{mx} / \mathrm{minitab} / 18 /$ help-and-how-to/quality-andprocess-improvement/measurement-systemanalysis/how-to/attribute-agreementanalysis/attribute-agreement-analysis/beforeyou-start/overview/

Romero, L. F., \& Romero, D. B. (2011). Estudio $\mathrm{R}$ y R por atributos de un proceso de inspección en el sector automotriz. Produção Em Foco, $1(1)$. https://doi.org/10.14521/P22375163.2011.0001.0010

Servizo Galego de Saúde. (2014). Epidat 4:Ayuda de concordancia y consistencia. recuperado de http://dxsp.sergas.essoporte.epidat@sergas.es 